\title{
SwiveLock Bone Anchor Stabilization of the Cranial Cruciate Ligament Deficient Stifle in Dogs: Clinical Outcome
}

\author{
Matthew Raske ${ }^{1}$, Don Hulse ${ }^{2}$ \\ ${ }^{1}$ Capital Area Veterinary Specialists, Austin, USA \\ ${ }^{2}$ Department of Veterinary Small Animal Clinical Sciences, College of Veterinary Medicine, \\ Texas A\&M University, College Station, USA \\ Email: matthew.raske@amcny.org
}

Received September 19, 2103; revised October 27, 2013; accepted November 6, 2013

Copyright (C) 2013 Matthew Raske, Don Hulse. This is an open access article distributed under the Creative Commons Attribution License, which permits unrestricted use, distribution, and reproduction in any medium, provided the original work is properly cited.

\begin{abstract}
A number of surgical procedures have been reported for the cranial cruciate ligament (CCL) deficient stifle in dogs. The objectives of this study were to report long-term outcomes in dogs with CCL deficient stifles stabilized with a knotless SwiveLock bone anchor preloaded with FiberTape and to report associated complications. Medical records were reviewed to identify dogs with unilateral CCL deficient stifles treated with the SwiveLock system $(n=41)$ between June 2008 and January 2012. Dogs were divided into three groups according to body weight to assess differences between owner assessed functional outcomes. Complications were recorded and a validated owner questionnaire was used to evaluate long-term outcomes at least 6 months postoperatively. Owner assessment prior to and after treatment with the SwiveLock system was statistically significant for each of the categories of the validated owner questionnaire. There was no significant difference among the weight groups for any of the preoperative and postoperative treatment owner questions. Complications requiring surgical revision occurred in three (7.3\%) dogs. Two had surgical site infections and one had a subsequent meniscal tear. The authors concluded that the SwiveLock system placed at near isometric sites is an effective surgical option for the treatment of dogs with CCL deficient stifles.
\end{abstract}

Keywords: Canine; Cranial Cruciate Ligament; FiberTape; Isometric Stabilization; SwiveLock

\section{Introduction}

Cranial cruciate ligament (CCL) injury is a primary cause of lameness in the canine, which leads to stifle instability and predisposes to degenerative changes [1]. There are numerous surgical options for treating the CCL deficient stifle, but one technique has not been proven to be superior. Popular methods for treating the CCL deficient stifle include extracapsular stabilization (ES) and tibial osteotomies that alter joint mechanics [2,3]. Extracapsular stabilization (ES) has been used for over the past half century to treat CCL injuries and has yielded good to excellent results $[3,4]$. Advantages of ES compared to other procedures include a better safety profile and a procedure that is less technically demanding [2].

The knotless SwiveLock anchor system ${ }^{1}$ is a stifle sta-

${ }^{1}$ SwiveLock Knotless Anchor System; Arthrex Vet Systems, Naples, Florida, USA. bilization technique that utilizes a bone anchor and $2 \mathrm{~mm}$ multifilament FiberTape ${ }^{2}$. This stabilizing suture is placed at near isometric points F2-T3 (the location is at the distal pole of the fabella just cranial to the margin of the articular cartilage on the lateral femoral condyle and at the bony prominence just caudal to the long digital extensor groove of the proximal tibia) allowing for minimal changes of suture tension through flexion/extension, while allowing for a more natural stifle movement [5]. Ultimately, the near isometric points reduce incidence of implant failure and may result in improved clinical outcomes [6].

The purpose of this manuscript is to report the long-term outcomes of dogs with CCL deficient stifles stabilized with a knotless SwiveLock bone anchor preloaded with $2 \mathrm{~mm}$ FiberTape placed at near isometric

\footnotetext{
${ }^{2}$ FiberTape; Arthrex Vet Systems, Naples, Florida, USA.
} 
sites.

\section{Materials and Methods}

Medical records of dogs with CCL injury treated with a knotless SwiveLock bone anchor preloaded with $2 \mathrm{~mm}$ FiberTape from June 2008-January 2012 were reviewed. Dogs that were at least 6 months post operative after stabilization were considered for inclusion. Dogs were excluded when additional orthopedic and/or neurological disorders were diagnosed before or after surgery, if the patient was deceased, or if owners could not be reached for follow up.

Data obtained from the medical records included signalment (breed, age, weight, and gender), affected limb, extent of CCL injury, meniscal damage, presence of cranial drawer at final clinical exam (7 - 8 week recheck), complications, and time to telephone or e-mail follow up.

\subsection{Pre- and Postoperative Care}

All dogs were premedicated with intramuscular (IM) acepromazine $^{3}(0.025 \mathrm{mg} / \mathrm{kg})$, hydromorphone ${ }^{4}(0.1 \mathrm{mg} / \mathrm{kg})$, and glycopyrrolate $(0.01 \mathrm{mg} / \mathrm{kg})$. General anesthesia was induced intravenously (IV) with propofol ${ }^{6}(5 \mathrm{mg} / \mathrm{kg}$ ) and maintained with isoflurane in oxygen. After induction, epidural analgesia was administered using preservativefree morphine ${ }^{7}(0.1 \mathrm{mg} / \mathrm{kg})$. Cefazolin ${ }^{8}(22 \mathrm{mg} / \mathrm{kg})$ was given IV as a perioperative antibiotic at the start of surgery and every 90 minutes as needed. An intra-articular morphine $^{9}(0.5 \mathrm{mg} / \mathrm{kg})$ block and bupivacaine ${ }^{10}(2.5-5.0$ $\mathrm{mg}$ ) local incisional block were administered postoperatively, as well as carprofen ${ }^{11}(4.4 \mathrm{mg} / \mathrm{kg})$ subcutaneously. Morphine $^{9}(0.5 \mathrm{mg} / \mathrm{kg})$ was administered IV every 4 hours for the following 24 hours as needed. Tramadol ${ }^{12}$ (3 - $5 \mathrm{mg} / \mathrm{kg}$, per os [PO]) was administered 12 hours postoperatively and then 4 times daily for 3 days. Carprofen ${ }^{11}(2.2 \mathrm{mg} / \mathrm{kg}, \mathrm{PO})$ was administered 24 hours postoperatively and then twice daily for 14 days and once daily for 7 days thereafter. Dogs were administered cephalexin $^{13}(22 \mathrm{mg} / \mathrm{kg}$ PO) twice daily for 7 days.

The limb affected with the CCL injury was treated by arthroscopic assisted isometric stabilization with the

\footnotetext{
${ }^{3}$ Acepromazine; Boehringer Ingelheim Vetmedica Inc., St. Joseph, Missouri, USA.

${ }^{4}$ Hydromorphone Hydrochloride, Baxter Healthcare, Deerfield, Illinois, USA.

${ }^{5}$ Glycopyrrolate, Baxter Healthcare, Deerfield, Illinois, USA.

${ }^{6}$ Propofol, Hospira Inc., Lake Forest, Illinois, USA.

${ }^{7}$ Duramorph, Baxter Healthcare, Deerfield, Illinois, USA.

${ }^{8}$ Cefazolin, West-Ward Injectables Inc., Eatontown, New Jersey, USA.

${ }^{9}$ Morphine Sulfate, Baxter Healthcare, Deerfield, Illinois, USA

${ }^{10}$ Bupivacaine Hydrochloride $0.25 \%$, Hospira Inc., Lake Forest, Illinois USA.

${ }^{11}$ Rimadyl, Pfizer Animal Health, New York, New York, USA.

${ }^{12}$ Tramadol Hydrochloride Tablets, Apotex Inc., Toronto, Ontario, Canada.

${ }^{13}$ Cephalexin Capsules, Novopharm, Scarborough, Ontario, Canada.
}

knotless SwiveLock bone anchor preloaded with $2 \mathrm{~mm}$ FiberTape as previously described by Hulse et al. [6]. The size of the SwiveLock used was subjectively determined by the body weight and activity level of the patient. Craniocaudal and mediolateral radiographs were performed postoperatively to assess isometric position by documenting anchor and tunnel position. All dogs were discharged with identical postoperative rehabilitation instructions that included short restricted activity on leash until the 7 - 8 week recheck. Physical therapy was recommended beginning 2 weeks after surgery and consisted of specific treatments including use of the underwater treadmill, balance board, and physioroll, as well as exercises prescribed for home. Dogs returned for final evaluation at $7-8$ weeks and the presence of cranial drawer (abnormal craniocaudal translation evaluated without sedation) and complications were recorded.

Function of the affected stifle was evaluated at least 6 months post operatively using a validated owner questionnaire [7]. Owners were contacted via telephone or e-mail (when available) to respond to the questionnaire, which included seven questions pertaining to their pet's function after CCL injury, but prior to stabilization surgery and the same seven questions pertaining to function after surgical repair and rehabilitation. A scale of $1-6$ was used for all questions, with 1 being the worst and 6 being the best.

\subsection{Statistical Analysis}

Dogs were grouped according to body weight to assess differences between owner assessed functional outcome. Dogs in group one were less than $15 \mathrm{~kg}$, dogs in group two were $15-30 \mathrm{~kg}$, and dogs in group three were greater than $30 \mathrm{~kg}$. Analyses for differences between owners' responses to the pre and post stabilization questionnaire were performed using the Wilcoxon signed- rank test. A Kruskal Wallis test was used to examine dif- ferences among the three weight groups. Significance was set at $\mathrm{P}$ $<0.05$.

\section{Results}

Forty-one out of forty-nine dogs treated with a knotless Swivelock bone anchor preloaded with $2 \mathrm{~mm}$ FiberTape met the inclusion criteria. One dog was excluded as it had additional orthopedic disorders at the time the owner was contacted to answer the validated questionnaire, two dogs were deceased at time of follow up, and five dogs were lost to follow up as their owners were unable to be contacted.

There were 25 spayed (61.0\%) and two intact females $(4.8 \%)$ and 14 neutered males $(34.1 \%)$. A variety of breeds were represented in the study: 12 mixed breeds (29.3\%), six American Staffordshire Terriers (14.6\%), 
five Old English Bulldogs (12.2\%), three each of Rottweilers and Golden Retrievers (7.3\%), two each of Boxers and Labradors (4.8\%) and one each of the following: Bichon Frise, Catahoula, Chihuahua, Greater Swiss Mountain Dog, Scottish Terrier, Shar Pei, Shetland Sheepdog, and Shih Tzu (2.4\%). The mean age was $7.0+/-3.7$ years (range $1-15.9 \mathrm{yr}$ ). There were five dogs in group one (less than $15 \mathrm{~kg}), 24 \mathrm{dogs}$ in group two $(15-30 \mathrm{~kg}$ ), and 12 dogs in group three (greater than $30 \mathrm{~kg}$ ). Dogs in group one had a mean body weight of $9.3+/-3.2 \mathrm{~kg}$ (range $5.5-12.7 \mathrm{~kg}$ ), dogs in group two had a mean body weight of $24.6+/-2.9 \mathrm{~kg}$ (range $19.5-29.1 \mathrm{~kg}$ ), and dogs in group three had a mean body weight of $38.2+/-$ $8.1 \mathrm{~kg}$ (range $30.0-52.4 \mathrm{~kg}$ ). There was no significant difference found among the different weight groups for any of the preoperative and postoperative treatment owner questions $(\mathrm{P}>0.05)$.

Of the 41 stifles, 21 were left $(51.2 \%)$ and 20 were right $(48.8 \%)$. All of the affected stifle joints were evaluated and treated by arthroscopy. Thirty-nine dogs (95.1\%) had a complete CCL tear, and two dogs $(4.9 \%)$ had a partial CCL injury. The medial meniscus was normal in $29(70.7 \%)$ of the stifles, of these $15(51.7 \%)$ had a meniscal release performed. A bucket handle tear was evident in 11 of the stifles and all subsequently had a partial meniscectomy performed. One of the stifles was found to have a previously released medial meniscus. The lateral meniscus was normal in 40 of the stifles and one had a radial tear. A $3.5 \mathrm{~mm}$ SwiveLock was used in the five dogs $(12.2 \%)$ in group one, a $4.75 \mathrm{~mm}$ SwiveLock was used in 28 dogs $(68.3 \%), 24$ that were in group two and four that were in group three, and a $5.5 \mathrm{~mm}$ SwiveLock was used in eight dogs $(19.5 \%)$, one that was in group two and seven that were in group three. In two stifles, a second FiberTape was placed at the F1-T3 isometric site. (5) The size of suture utilized and the decision to place a second FiberTape was subjectively determined based on the weight and activity level of the patient.

Three $(7.3 \%)$ out of the 41 cases experienced major complications. Two of the cases involved infections that required surgical removal of the implants and one was a subsequent meniscal tear. No minor complications were noted at the 4 week or 7 - 8 week recheck.

Presence of cranial drawer in millimeters was subjectively assessed by the same observer (DH) on physical examination without sedation and recorded at the final exam (7 - 8 weeks post operative period) Thirty-six (87.8\%) of the affected stifles presented with $0-3 \mathrm{~mm}$ of cranial drawer at $7-8$ weeks. The remaining five (12.2\%) were subjectively assessed to have $4-6 \mathrm{~mm}$.

Mean time from surgery to owners answering the validated questionnaire was 16.5 months (range 7 to 47 months). The mean owner responses to questions pertaining to pet's clinical function after injury, but prior to stabilization and mean responses to questions pertaining to pet's clinical function after stabilization with the knotless SwiveLock system are reported in Figure 1. The surgical intervention with the SwiveLock system did elicit statistically significant changes for all seven of the preoperative and postoperative stabilization treatment owner questions $(\mathrm{P}<0.001)$.

\section{Discussion}

The knotless SwiveLock system successfully maintained stability of the CCL deficient stifle through $7-8$ weeks following surgery and achieved good short to long-term clinical function as assessed by a subjective owner questionnaire using a categorical scale. Owners reported good to excellent clinical function based on the questionnaire. Scores in all three of the weight groups were improved following surgery as compared to pre stabilization values. This clinical assessment supports in vitro studies that demonstrate the SwiveLock system to have the least elongation failure and least peak to peak elongation when compared to other ES procedures [8].

Three cases $(7.3 \%)$ with major complications were identified. All three were considered major complications, as they required additional surgical intervention. We did not include the five cases that were subjectively assessed to have $4-6 \mathrm{~mm}$ of cranial drawer as complications, as they did not present with clinical dysfunction and did not require further surgical intervention.

Surgical site infections (SSIs) accounted for two of three complications $(4.9 \%)$. This reported incidence of SSIs falls within the expected infection rate of $2.5 \%$ to $5.8 \%$ that has been reported for most clean veterinary medicine surgical procedures and is also compatible with incidence of SSIs following ES stabilization reported in previous studies [9-11]. Although SSIs cannot be eliminated completely, preventative measures reduce their incidence. An antimicrobial incise drape ${ }^{14}$ was used to decrease skin flora contact and contamination of the FiberTape, although this practice is of questionable benefit in reducing contamination in canine surgical wounds [12]. FiberTape is an integral part of this procedure in that it is stronger, elongates less than other materials, has greater yield loads, and greater load to failure [13]. When placed at near isometric points less stress is reported in the suture tension [6]. We feel these advantages outweigh the negative association of multifilament suture material with increased SSI incidence [14]. Bacterial culture and antimicrobial sensitivity testing were used to confirm the presence of a bacterial infection and to determine antimicrobial sensitivity. One of the two dogs developed an infection 4 weeks post op. The culture revealed the presence of a Methicillin-resistant Staphylococcus pseudo-

\footnotetext{
${ }^{14}$ Ioban, 3M, Flemington, New Jersey, USA.
} 


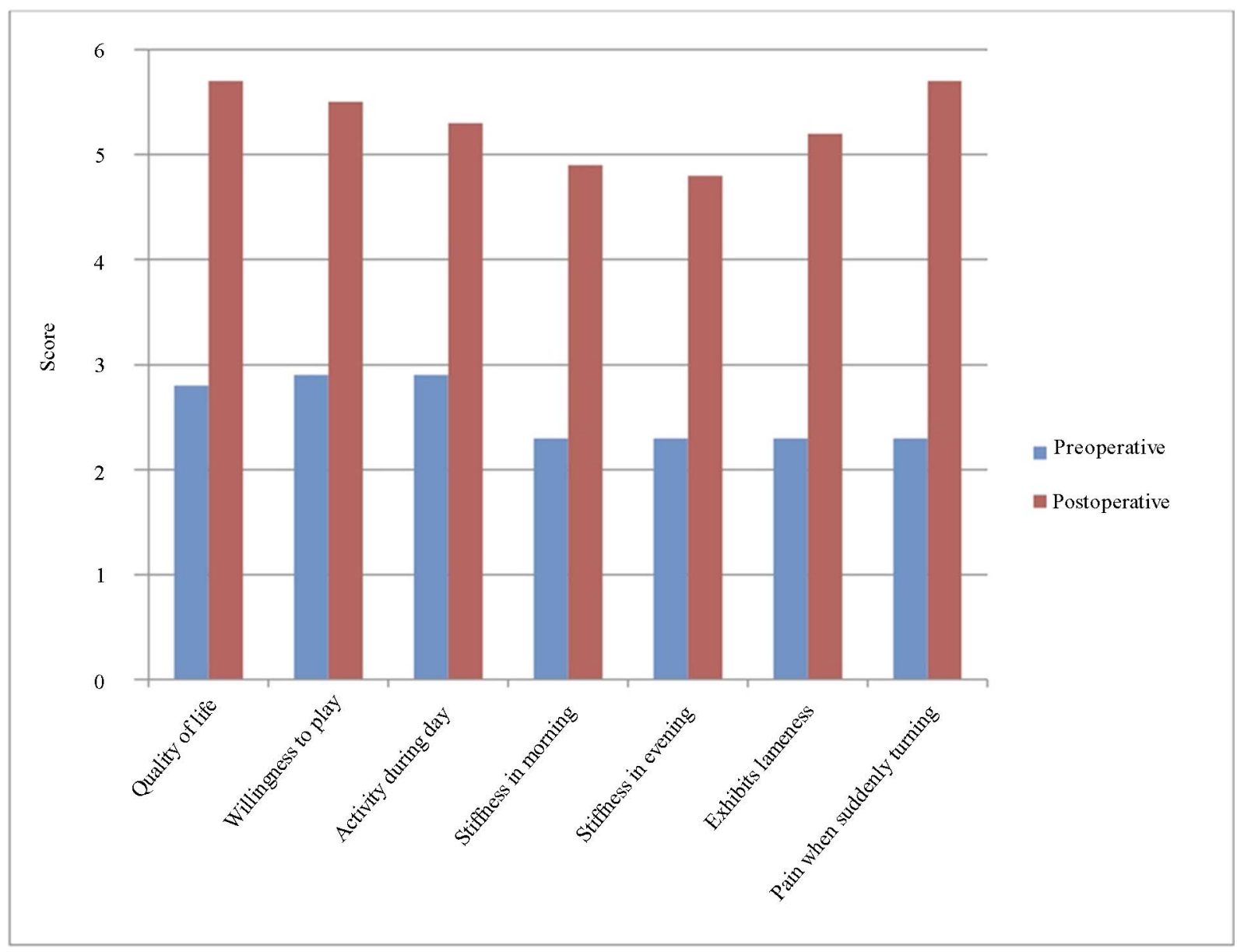

Figure 1. Validated questions posed to owners regarding pet's condition after injury, but prior to stabilization with a knotless SwiveLock system (blue) and after stabilization (red).

intermedius (MRSP). The dog was treated based on antimicrobial sensitivity results and the SwiveLock bone anchor with FiberTape was subsequently removed. The second dog developed a polymicrobial infection 6 weeks following surgery. Cultures revealed Staphylococcus aureus and Klebsiella spp. and the dog was treated for 4 weeks based on sensitivity results. Following treatment, the SwiveLock anchor and FiberTape were removed. In both cases, owner responses were good to excellent for all questions answered at 8 and 41 months, respectively.

The third complication was a late onset meniscal tear. The dog had progressed well and achieved a level of excellent clinical function until 6 months postoperative when the patient became suddenly lame in the operated limb. Arthroscopic $2^{\text {nd }}$ look examination revealed a medial meniscal tear, which was treated with partial meniscectomy. At the time of initial stabilization, the dog had a normal medial meniscus and a release was not performed. Following the $2^{\text {nd }}$ surgery, the dog made a full recovery and was clinically sound at recheck one month after the partial meniscectomy.

Our complication rate of $7.3 \%$ is consistent with other studies reporting complication rates after treatment with extracapsular stabilization. Cook et al. [15] reported a $12.5 \%$ major complication rate for TightRope ${ }^{15}$ procedure. Casale and McCarthy [16] found a 17.4\% complication rate associated with lateral fabellotibial suture procedures. There was no incidence of bone anchor pull out which is, in contrast to a $21 \%$ of anchor failure after a lateral suture anchor procedure in a separate study [17].

The authors recognize several limitations to this study. We did not track the progression of osteoarthritis on radiographs or $2^{\text {nd }}$ look arthroscopy when appropriate and the presence of cranial drawer was not assessed while the dog was sedated. Other limitations include the small population size and the retrospective nature of the study. Another limitation of this study is the potential for a caregiver placebo effect. In a study evaluating patient response to osteoarthritis, treatment it concluded that caregiver placebo effect should be considered when determining patient response to treatment based on owner and veterinarian subjective assessment. [18] Functional

\footnotetext{
${ }^{15}$ TightRope; Arthrex Vet Systems, Naples, Florida, USA.
} 
outcome was based on a subjective, client answered questionnaire. The questionnaire has been found to be repeatable and valid for assessing lameness in dogs. [6]

\section{Conclusion}

The knotless SwiveLock bone anchor preloaded with 2 mm FiberTape provides good to excellent long-term clinical function outcomes and a low complication rate in dogs treated for CCL deficient stifles.

\section{Acknowledgements}

We would like to thank Dr. Abigail D. Mariano for assistance with statistical analysis.

\section{REFERENCES}

[1] D. L. Piermattei, F. L. Gretchen and C. E. DeCamp, "The Stifle Joint," In: D. L. Piermattei, F. L. Gretchen and C. E. DeCamp, Eds., Brinker, Piermattei, and Flo's Handbook of Small Animal Orthopedics and Fracture Repair, Elsevier Inc., St. Louis, 2006, pp. 562-632.

[2] J. L. Cook, "Extracapsular Stabilization,” In: P. Muir, Ed., Advances in the Canine Cranial Cruciate Ligament, Wiley-Blackwell, Hoboken, 2010, pp. 163-167.

[3] S. E. Kim, A. Pozzi, M. P. Kowaleski, et al., "Tibial Osteotomies for Cranial Cruciate Ligament Insufficiency in Dogs," Veterinary Surgery, Vol. 37, No. 2, 2008, pp. 111125. http://dx.doi.org/10.1111/j.1532-950X.2007.00361.x

[4] H. S. Mullen and D. T. Matthiesen, "Complications of Transposition of the Fibular Head for Stabilization of the Cranial Cruciate-Deficient Stifle in Dogs: 80 Cases (19821986)," Journal of the American Animal Hospital Association, Vol. 195, No. 9, 1989, pp. 1267-1271.

[5] D. Hulse, W. Hyman, B. Beale, et al., "Determination of Isometric Points for Placement of a Lateral Suture in Treatment of the Cranial Cruciate Ligament Deficient Stifle," Veterinary and Comparative Orthopaedics and Traumatology, Vol. 23, No. 3, 2010, pp. 163-167.

[6] D. Hulse, B. Saunders, B. Beale, et al., "Extra-Articular Stabilization of the Cranial Cruciate Deficient Stifle with Anchor Systems," Tierärztliche Praxis Kleintiere, Vol. 39, No. 5, 2011, pp. 363-367.

[7] J. T. Hudson, M. R. Slater, L. Taylor, et al., "Assessing Repeat Ability and Validity of a Visual Analogue Scale Questionnaire for Use in Assessing Pain and Lameness in Dogs," American Journal of Veterinary Research, Vol. 65, No. 12, 2004, pp. 1634-1643. http://dx.doi.org/10.2460/ajvr.2004.65.1634

[8] C. J. Choate, A. Pozzi, D. D. Lewis, et al., "Mechanical Comparison of Lateral Circumfabellar Suture, Tightrope $\mathrm{ccl}$, and Swivelock Bone Anchor for Extracapsular Stabilization of the Cranial Cruciate Ligament-Deficient Stifle in Dogs," Proceed 32nd Annual Conference of the Veterinary Orthopedic Society, Snowmass, 2005, pp. 9-10.

[9] S. Eugster, P. Schawalder, F. Gaschen, et al., "A Prospective Study of Postoperative Surgical Site Infections in Dogs and Cats," Veterinary Surgery, Vol. 33, No. 5, 2004, pp. 542-550.

http://dx.doi.org/10.1111/j.1532-950X.2004.04076.x

[10] T. N. Frey, M. G. Hoelzler, T. D. Scavelli, et al., "Risk Factors for Surgical Site Infection-Inflammation in Dogs Undergoing Surgery for Rupture of the Cranial Cruciate Ligament: 902 Cases (2005-2006)," Journal of the American Veterinary Medical Association, Vol. 236, No. 1, 2010, pp. 88-94.

http://dx.doi.org/10.2460/javma.236.1.88

[11] P. B. Vasseur, J. Levy, E. Dowd, et al., "Surgical Wound Infection Rates in Dogs and Cats Data from a Teaching Hospital," Veterinary Surgery, Vol. 17, No. 2, 1988, pp. 60-64.

http://dx.doi.org/10.1111/j.1532-950X.1988.tb00278.x

[12] L. J. Owen, J. A. Gines, T. G. Knowles, et al., "Efficacy of Adhesive Incise Drapes in Preventing Bacterial Contamination of Clean Canine Surgical Wounds," Veterinary Surgery, Vol. 38, No. 6, 2009, pp. 732-737. http://dx.doi.org/10.1111/j.1532-950X.2009.00537.x

[13] N. D. Rose, D. Goerke, R. B. Evans, et al., "Mechanical Testing of Orthopedic Suture Material Used for ExtraArticular Stabilization of Canine Cruciate-Ligament Deficient Stifles," Veterinary Surgery, Vol. 41, No. 2, 2012, pp. 266-272.

[14] M. L. Dulisch, "Suture Reaction Following Extra-Articular Stifle Stabilization in the Dog-Part I: A Retrospective Study of 161 Stifles," Journal of the American Veterinary Medical Association, Vol. 17, 1981, pp. 569571.

[15] J. L. Cook, J. K. Luther, J. Beetem, et al., "Clinical Comparison of a Novel Extracapsular Stabilization Procedure and Tibial Plateau Leveling Osteotomy for Treatment of Cranial Cruciate Ligament Deficiency in Dogs," Veterinary Surgery, Vol. 39, No. 3, 2010, pp. 315-323. http://dx.doi.org/10.1111/j.1532-950X.2010.00658.x

[16] S. A. Casale and R. J. McCarthy, "Complications Associated with Lateral Fabellotibial Suture Surgery for Cranial Cruciate Ligament Injury in Dogs: 363 Cases (19972005)," Journal of the American Veterinary Medical Association, Vol. 234, No. 2, 2009, pp. 229-235.

[17] L. Guenego, A. Zahara, A. Madelenat, et al., "Cranial Cruciate Ligament Rupture in Large and Giant Dogs. A retrospective Evaluation of a Modified Lateral Extracapsular Stabilization," Veterinary and Comparative Orthopaedics and Traumatology, Vol. 20, No. 1, 2007, pp. 43-50.

[18] M. G. Conzemius and R. B. Evans, "Caregiver Placebo Effect for Dogs with Lameness from Osteoarthritis," Journal of the American Veterinary Medical Association, Vol. 241, No. 10, 2012, pp. 1314-1319.

http://dx.doi.org/10.2460/javma.241.10.1314 